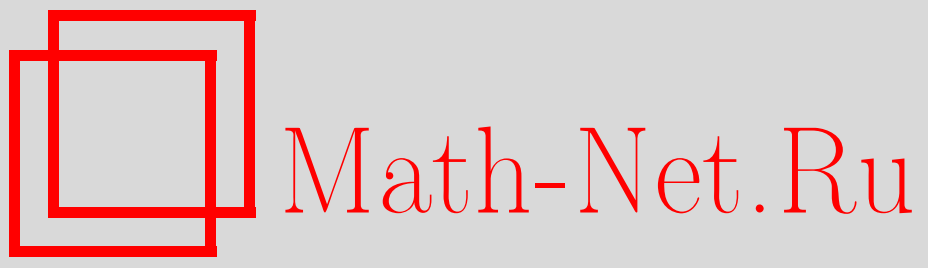

И. А. Ибрагимов, Н. В. Смородина, М. М. Фаддеев, Предельные теоремы о сходимости математических ожиданий функционалов от сумм независимых случайных величин к решениям начально-краевых задач, Теория вероятн. и ее примен., 2014, том 59, выпуск 2, 233-251

DOI: https://doi.org/10.4213/tvp4564

Использование Общероссийского математического портала Math-Net.Ru подразумевает, что вы прочитали и согласны с пользовательским соглашением http://www . mathnet.ru/rus/agreement

Параметры загрузки:

IP : 52.6 .47 .48

26 апреля 2023 г., 16:56:19

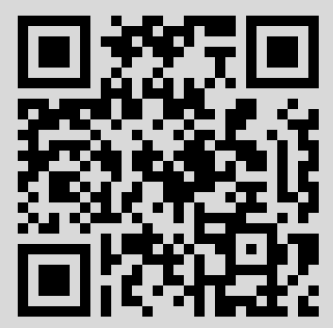




\title{
ПРЕДЕЛЬНЫЕ ТЕОРЕМЫ О СХОДИМОСТИ МАТЕМАТИЧЕСКИХ ОЖИДАНИЙ ФУНКЦИОНАЛОВ ОТ СУММ НЕЗАВИСИМЫХ СЛУЧАЙНЫХ ВЕЛИЧИН К РЕШЕНИЯМ НАЧАЛЬНО-КРАЕВЫХ ЗАДАЧ ${ }^{1)}$
}

\begin{abstract}
В работе доказываются предельные теоремы о сходимости математических ожиданий функционалов от некоторых случайных блужданий к решению начально-краевой задачи для уравнения $\partial u / \partial t=\left(\sigma^{2} / 2\right) \Delta u=0$, где $\sigma$ - комплексный параметр, удовлетворяющий условию $\operatorname{Re} \sigma^{2} \geqslant 0$.

Ключевые слова и фразы: начально-краевая задача, предельные теоремы, мера Фейнмана, псевдопроцессы.
\end{abstract}

1. Постановка задачи. Пусть $\sigma$ - комплексное число, удовлетворяющее условию $\operatorname{Re} \sigma^{2} \geqslant 0$. Рассмотрим уравнение

$$
\frac{\partial u}{\partial t}=\frac{\sigma^{2}}{2} \Delta u
$$

(здесь $u=u(t, x), t \geqslant 0, x \in \mathbf{R}^{d}$ ). Если $\sigma$ - вещественное число, то уравнение (1) — это уравнение теплопроводности, а случай $\operatorname{Re} \sigma^{2}=0$ соответствует уравнению Шрёдингера. Таким образом, параметр $\sigma$ связывает в единое семейство уравнения теплопроводности и Шрёдингера.

Далее, пусть $D$ - ограниченная область в $\mathbf{R}^{d}, d \geqslant 2$, с гладкой границей $\partial D$. Для уравнения (1) мы рассмотрим начально-краевую задачу с граничными условиями Дирихле

$$
u(0, x)=\varphi(x),\left.\quad u\right|_{\partial D}=0 .
$$

*ПОМИ РАН, Санкт-Петербургский государственный университет, Санкт-Петербург, Россия; e-mail: ibr32@pdmi.ras.ru

** Санкт-Петербургский государственный университет, Санкт-Петербург, Россия; e-mail: nsmorodna@yahoo.com; mmf@ns2691.spb.ru,mmfaddeev@gmail.com

1) Работа первого автора выполнена при поддержке РФФИ (грант № 11-01-00577а), программы «Ведущие научные школы» (грант НШ-1216.2012.1) и программы фундаментальных исследований РАН «Современные проблемы математики». Работа второго автора выполнена при поддержке РФФИ (грант № 12-01-00487а) и ФЦП Минобрнауки (соглашение № 8501 от 07.09.2012). Работа третьего автора выполнена при поддержке РФФИ (грант № 12-01-00215а), ФЦП Минобрнауки (соглашение № 8501 от 07.09.2012). 
В случае, когда $\sigma$ - вещественное число, решение начально-краевой задачи (1), (2) допускает вероятностное представление в виде математического ожидания функционала от винеровского процесса, остановленного в момент первого достижения границы $\partial D$ области $D$. Именно, для каждого $x \in D$ определим процесс $\xi_{x}(t), t \geqslant 0$, полагая

$$
\xi_{x}(t)=x+\sigma w(t)
$$

где $w(t)=\left(w_{1}(t), \ldots, w_{d}(t)\right)$ - вектор, координаты которого суть независимые стандартные винеровские процессы, стартующие из нуля. Через $\tau$ обозначим момент первого достижения процессом $\xi_{x}(t)$ границы области $D$ и определим остановленный в этот момент процесс $\widetilde{\xi}_{x}(t)$, полагая

$$
\widetilde{\xi}_{x}(t)=\xi_{x}(t \wedge \tau) .
$$

Известно (см. [1], [2]), что для всякой непрерывной на $D$ функции $\varphi$ функция

$$
u(t, x)=\operatorname{E} \varphi\left(\widetilde{\xi}_{x}(t)\right)
$$

является решением начально-краевой задачи $(1),(2)$, что означает, что семейство одномерных распределений процесса $\widetilde{\xi}_{x}(t)$ есть фундаментальное решение (1),(2). Представление (4) решения начально-краевой задачи называют еще представлением решения в виде функционального интеграла (подробнее про функциональный интеграл см. [6], [7]).

В качестве вероятностного пространства в (4) можно взять пространство $C_{0}\left([0, T], \mathbf{R}^{d}\right)$ непрерывных функций $h:[0, T] \rightarrow \mathbf{R}^{d}$ таких, что $h(0)=0$, с винеровской мерой $P_{\sigma}$. Мера $P_{\sigma}$ однозначно продолжается с алгебры цилиндрических множеств на $\sigma$-алгебру борелевских множеств $C_{0}\left([0, T], \mathbf{R}^{d}\right)$. При этом для каждого набора моментов времени $0<t_{1}<\cdots<t_{n} \leqslant T$ и каждого борелевского множества $A \subset\left(\mathbf{R}^{d}\right)^{n}$ мера $P_{\sigma}\left(U_{t_{1}, \ldots, t_{n}, A}\right)$ цилиндрического множества $U_{t_{1}, \ldots, t_{n}, A}$ вида

$$
U_{t_{1}, \ldots, t_{n}, A}=\left\{h \in C_{0}[0, T]:\left(h\left(t_{1}\right), h\left(t_{2}\right), \ldots, h\left(t_{n}\right)\right) \in A\right\}
$$

равна

$$
\frac{1}{\sigma^{n}(2 \pi)^{n d / 2} \prod_{j=1}^{n}\left(t_{j}-t_{j-1}\right)^{1 / 2}} \int_{A} \exp \left\{-\frac{1}{2 \sigma^{2}} \sum_{j=1}^{n} \frac{\left\|x_{j}-x_{j-1}\right\|^{2}}{t_{j}-t_{j-1}}\right\} d x_{1} \cdots d x_{n} .
$$

Известно [3], что в случае $\operatorname{Im} \sigma \neq 0$ комплексная мера $P_{\sigma}$, которая на цилиндрических множествах задается формулой (5), не продолжается на $\sigma$-алгебру борелевских множеств $C_{0}\left([0, T], \mathbf{R}^{d}\right)$, что приводит к невозможности реализовать в этом случае функциональный интеграл как интеграл по мере в пространстве траекторий. Такие объекты, которые порождают в пространстве траекторий (невероятностную) меру 
только на алгебре цилиндрических множеств, носят в литературе название псевдопроцессов. Имеется большое количество результатов о распределении функционалов от псевдопроцессов (см., например, [4], [5]), в частности, имеются результаты, показывающие, что образ конечноаддитивной меры под действием достаточно гладкого отображения может оказаться уже счетно-аддитивной мерой.

Наиболее известен и изучен частный случай этой конструкции, когда $\operatorname{Re} \sigma^{2}=0$, т.е. когда уравнение (1) является уравнением Шрёдингера. В этом случае конечно-аддитивная мера, которая появляется в представлении решения в виде функционального интеграла, носит название меры Фейнмана. Соответствующий интеграл (интеграл Фейнмана) определяется через предел по измельчающейся последовательности разбиений интервала $[0, T]$. Следует отметить, что данный подход (это же относится и ко всей теории псевдопроцессов) не является в обычном смысле вероятностным, так как в нем отсутствует само понятие вероятностного пространства. Обзор публикаций, посвященных различным подходам к построению интеграла Фейнмана, можно найти в [6]-[10].

Далее, в работе авторов [11] был предложен метод аппроксимации решения одномерной $(d=1)$ начально-краевой задачи $(1),(2)$ математическими ожиданиями функционалов от последовательности сложных пуассоновских процессов, опирающийся на использование традиционных методов теории вероятностей. В этой работе использовались сложные пуассоновские процессы специального вида, у которых распределение величины скачка является гауссовским. Далее в работе [12] этим же методом была построена вероятностная аппроксимация решения начальнокраевой задачи (1), (2) уже в пространстве произвольной размерности, при этом, так же как и в [11], использовались сложные пуассоновские процессы с гауссовским распределением величины скачка.

Предложенный в [11] подход основан на использовании формулы (4), но для комплексного $\sigma$. Это невозможно сделать непосредственно, так как, во-первых, в этом случае процесс (3) становится комплекснозначным и, соответственно, не имеет смысла говорить о моменте первого достижения границы, а во-вторых, не ясно, как подставить комплексную величину (вектор с комплексными координатами) в аргумент функции $\varphi$, которая определена только на $D$.

Для решения первой проблемы (с моментом остановки) в [11], [12] использовался прием, связанный с продолжением начального данного $\varphi$ до функции $\widetilde{\varphi}$, заданной уже на всем $\mathbf{R}^{d}$, а для того, чтобы подставить в аргумент функции $\widetilde{\varphi}$ комплексный $d$-мерный вектор, функция $\widetilde{\varphi}$ предварительно аппроксимировалась тригонометрическим полиномом.

Далее, в работе [13] рассматривался вопрос вероятностной аппроксимации решения задачи Коши для уравнения (1). Именно, рассматри- 
валось уравнение (1) во всем $\mathbf{R}^{d}$ с начальным данным

$$
u(0, x)=\varphi(x)
$$

причем предполагалось, что $\operatorname{Re} \sigma^{2} \geqslant 0$, а начальное данное $\varphi$ принадлежит соболевскому классу $W_{2}^{l}$ при некотором $l \geqslant 4$.

Чтобы сформулировать основной результат работы [13], нам потребуется ввести дополнительные обозначения. Для $t \geqslant 0$ через $P^{t}$ обозначим операторную экспоненту $e^{\left(t \sigma^{2} / 2\right) \Delta}$. По определению оператор $P^{t}$ отображает функцию $\varphi$ в решение $u(t, \cdot)$ задачи Коши $(1),(6)$. Семейство операторов $P^{t}$ образует полугруппу, т.е. $P^{t+s}=P^{t} P^{s}$ для всех $t, s \geqslant 0$. Решение задачи Коши (1), (6) представляется в виде свертки с фундаментальным решением, именно,

$$
u(t, x)=P^{t} \varphi(x)=\frac{1}{\sigma^{d}(2 \pi)^{d / 2}} \int_{\mathbf{R}^{d}} \varphi(x+y) e^{-\|y\|^{2} /\left(2 \sigma^{2}\right)} d y .
$$

Соответствующее фундаментальное решение является вероятностной мерой только в случае вещественного $\sigma$, и в этом случае решение задачи Коши имеет вероятностное представление вида

$$
u(t, x)=\mathbf{E} \varphi(x+\sigma \sqrt{t} \xi)=\mathbf{E} \varphi(x+\sigma w(t)),
$$

где $\xi$ - гауссовская $\mathbf{R}^{d}$-значная случайная величина с единичной матрицей ковариаций, $w(t)$ - стандартный $d$-мерный винеровский процесс. В случае комплексного $\sigma$ формула (8) формально не имеет смысла, так как функция $\varphi$ является функцией вещественного аргумента, но, тем не менее, она может быть использована для вероятностной аппроксимации решения задачи Коши (1), (6). Поясним последнее простым одномерным $(d=1)$ примером.

Рассмотрим для комплексного $\sigma, \operatorname{Re} \sigma^{2} \geqslant 0$, интеграл

$$
\frac{1}{\sigma \sqrt{2 \pi}} \int_{\mathbf{R}} \varphi(x+y) e^{-y^{2} /\left(2 \sigma^{2}\right)} d y .
$$

Этот интеграл для невещественного $\sigma$ не имеет явного вероятностного смысла, так как не является математическим ожиданием вида $\mathbf{E} \varphi(\xi)$ ни для какой случайной величины $\xi$.

Предположим теперь дополнительно, что функция $\varphi$ ограничена на $\mathbf{R}$ и одновременно продолжается до целой аналитической функции экспоненциального типа. Тогда, поворачивая контур интегрирования, мы получаем

$$
\frac{1}{\sigma \sqrt{2 \pi}} \int_{\mathbf{R}} \varphi(x+y) e^{-y^{2} /\left(2 \sigma^{2}\right)} d y=\frac{1}{\sqrt{2 \pi}} \int_{\mathbf{R}} \varphi(x+\sigma y) e^{-y^{2} / 2} d y .
$$


Последнее выражение представляет собой математическое ожидание

$$
\mathbf{E} \psi(\eta),
$$

где $\eta$ - стандартная нормальная случайная величина, а $\psi$ - функция, которая представляет собой сужение на прямую $x+\sigma t, t \in \mathbf{R}$, аналитического продолжения функции $\varphi$.

Таким образом, мы представили (9) в виде математического ожидания, но уже от другой функции. Для примера, если мы $\varphi(y)=\sin y$ и $\sigma=e^{i \pi / 4}$ то, как легко сосчитать,

$$
\psi(y)=\sin \left(x+\frac{y}{\sqrt{2}}\right) \cosh \frac{y}{\sqrt{2}}+i \cos \left(x+\frac{y}{\sqrt{2}}\right) \sinh \frac{y}{\sqrt{2}} .
$$

Итак, для вышеуказанного класса функций $\varphi$ мы имеем

$$
\frac{1}{\sigma \sqrt{2 \pi}} \int_{\mathbf{R}} \varphi(x+y) e^{-y^{2} /\left(2 \sigma^{2}\right)} d y=\mathbf{E} \psi(\eta)
$$

что позволяет дать вероятностную трактовку интегралу (9).

Таким образом, для невещественных $\sigma$ вероятностное представление интеграла (9) имеет место, но для очень узкого класса функций $\varphi$. Вероятностная аппроксимация решения может быть получена таким образом при помощи подходящей аппроксимации начального данного $\varphi$ целой аналитической функцией.

Следующий вопрос, который в данном случае естественно возникает, это вопрос о том, будет ли иметь место сходимость к решению задачи Коши, если мы не только будем аппроксимировать начальное данное целой функцией, но и одновременно заменять нормальную случайную величину какой-то ее аппроксимацией. В силу центральной предельной теоремы естественно в качестве такой аппроксимации выбрать нормированную сумму независимых случайных величин. Приведем здесь соответствующий результат из [13].

Пусть $\left\{\xi_{j}\right\}_{j=1}^{\infty}$ - последовательность независимых одинаково распределенных $\mathbf{R}^{d}$-значных случайных величин с симметричным распределением $\mathscr{P}$ каждая. Предполагается, что случайные величины имеют единичную матрицу ковариаций и конечный экспоненциальный момент, т.е. для некоторого $\gamma>0$

$$
\mathbf{E} e^{\gamma\|\xi\|}<\infty .
$$

Далее, пусть $\eta(t), t \in[0, \infty),-$ независимый от последовательности $\left\{\xi_{j}\right\}$ пуассоновский процесс с параметром единица. Для каждого натурального $n$ определим случайный процесс $\zeta_{n}(t), t \in[0, T]$, полагая

$$
\zeta_{n}(t)=\frac{1}{\sqrt{n}} \sum_{j=1}^{\eta(n t)} \xi_{j} .
$$


$\mathrm{C}$ помощью процессов $\zeta_{n}(t)$ построим последовательность полугрупп операторов, аппроксимирующую полугруппу $P^{t}$. С этой целью для каждой (комплекснозначной) функции $\varphi \in L_{2}\left(\mathbf{R}^{d}\right)$ и числа $M>0$ определим функцию $\varphi_{M}$, полагая

$$
\varphi_{M}(x)=\frac{1}{(2 \pi)^{d}} \int_{\|p\| \leqslant M} \widehat{\varphi}(p) e^{-i(p, x)} d p,
$$

где, как обычно, $\widehat{\varphi}$ обозначает преобразование Фурье функции $\varphi$. Отметим, что при всех $M>0$ функция $\varphi_{M}$ (как функция $x \in \mathbf{C}^{d}$ ) является целой аналитической функцией экспоненциального типа. Далее число $M$ выбирается в зависимости от $n, M=M(n)$.

Для натуральных $n$ определим полугруппу операторов $P_{n}^{t}$, полагая для комплекснозначной $\varphi \in L_{2}\left(\mathbf{R}^{d}\right)$ и $x \in \mathbf{R}^{d}$

$$
P_{n}^{t} \varphi(x)=\mathbf{E} \varphi_{M}\left(x+\sigma \zeta_{n}(t)\right)
$$

Справедливо следующее утверждение [13].

Теорема 1. Пусть $\varphi \in W_{2}^{2 l+4}\left(\mathbf{R}^{d}\right), l \geqslant 0, M(n)=n^{1 / 4}$. Тогда

$$
\sup _{t \in[0, T]}\left\|P_{n}^{t} \varphi-P^{t} \varphi\right\|_{W_{2}^{2 l}\left(\mathbf{R}^{d}\right)} \leqslant C \frac{\|\varphi\|_{W_{2}^{2 l+4}\left(\mathbf{R}^{d}\right)}}{n},
$$

әде $C=C(l, d, T)$.

В настоящей работе мы докажем предельные теоремы, аналогичные теореме 1, с оценкой скорости сходимости в соболевских метриках, но не для задачи Коши, а для начально-краевой задачи (1), (2).

2. Основные обозначения. Пусть $\|\cdot\|$ обозначает евклидову норму в пространстве $\mathbf{R}^{d}$. Встречающиеся в выкладках константы обычно обозначаются буквой $C$, одна и та же буква может обозначать разные константы.

Через $W_{2}^{k}(D)$ мы будем обозначать соболевское пространство функций, определенных на $D$ и имеющих квадратично суммируемые обобщенные производные до порядка $k$ включительно. Через $\stackrel{\circ}{W}_{2}^{k}(D)$ мы обозначаем подпространство пространства $W_{2}^{k}(D)$, состоящее из тех элементов последнего, которые допускают продолжение нулем за пределы области $D$ до элементов пространства $W_{2}^{k}\left(\mathbf{R}^{d}\right)$. Теория пространств Соболева подробно изложена в [16], [18], [19].

3. Задача Дирихле в кубе. В этом пункте мы предположим, что область $D$ - это куб в $\mathbf{R}^{d}$. Для удобства будем считать, что это куб $[0, \pi]^{d}$, общий случай сводится к этому с помощью аффинного преобразования. Мы всегда будем предполагать, что (комплекснозначная) функция $\varphi$ (начальное условие задачи $(1),(2))$ принадлежит классу $L_{2}(D)$. 
Для $t \geqslant 0$ через $P^{t}$ обозначим операторную экспоненту $e^{\left(t \sigma^{2} / 2\right) \Delta_{\mathscr{D}}}$, где $\Delta_{\mathscr{D}}$ - самосопряженный оператор в пространстве $L_{2}(D)$, заданный на области определения $W_{2}^{2,0}(D)$, состоящей из элементов пространства $W_{2}^{2}(D)$, обращающихся в нуль на границе куба, и действующий как оператор Лапласа.

По определению оператор $P^{t}$ отображает функцию $\varphi$ в решение $u(t, \cdot)$ начально-краевой задачи $(1),(2)$. Семейство операторов $P^{t}$ образует полугруппу (т.е. $P^{t+s}=P^{t} P^{s}$ для всех $t, s \geqslant 0$ ).

Выпишем явное выражение для полугруппы $P^{t}$ в виде, удобном для последующих оценок. Для каждой функции $\varphi \in L_{2}(D)$ через $\varphi^{\text {odd }}$ обозначим ее нечетное (по каждой переменной) продолжение с $[0, \pi]^{d}$ на $[-\pi, \pi]^{d}$. Разложим функцию $\varphi^{\text {odd }}$ в ряд Фурье:

$$
\varphi^{\mathrm{odd}}(x)=\sum_{m \in \mathbf{Z}^{d}} B_{m} e^{i(m, x)} .
$$

Используя метод разделения переменных, получаем

$$
P^{t} \varphi(x)=\sum_{m \in \mathbf{Z}^{d}} B_{m} e^{-\sigma^{2} t\|m\|^{2} / 2} e^{i(m, x)} .
$$

Отметим, что из условия $\operatorname{Re} \sigma^{2} \geqslant 0$ следует, что

$$
\left|e^{-\sigma^{2} t\|m\|^{2} / 2}\right| \leqslant 1
$$

Следующим шагом с помощью последовательности случайных процессов (12) построим последовательность полугрупп операторов, аппроксимирующую полугруппу $P^{t}$. Введем необходимые обозначения. Для каждого $M>0$ через $\mathscr{H}_{M}$ обозначим проектор в $L_{2}\left([-\pi, \pi]^{d}\right)$ на линейное подпространство $\mathscr{L}\left(e_{m},\|m\| \leqslant M\right)$, где

$$
e_{m}(x)=e^{i(m, x)}
$$

При этом функция

$$
\varphi_{M}(x)=\mathscr{H}_{M} \varphi^{\text {odd }}(x)=\sum_{\|m\| \leqslant M} B_{m} e^{i(m, x)}
$$

является тригонометрическим полиномом и, соответственно, определена на всем $\mathbf{C}^{d}$. Далее число $M$ мы будем выбирать в зависимости от $n$, $M=M(n)$.

Для натуральных $n$ определим полугруппу операторов $P_{n}^{t}$, полагая для комплекснозначной $\varphi \in L_{2}(D)$ и $x \in \mathbf{R}^{d}$

$$
P_{n}^{t} \varphi(x)=\mathbf{E} \varphi_{M}\left(x+\sigma \zeta_{n}(t)\right)
$$

(процесс $\zeta_{n}(t)$ определен в (12)). 
Для каждого $l=0,1,2 \ldots$ через $H_{D}^{2 l}$ обозначим пространство функций $\varphi:[0, \pi]^{d} \rightarrow \mathbf{C}$ таких, что сходится ряд $\sum_{m \in \mathbf{Z}^{d}}\left|B_{m}\right|^{2}\|m\|^{4 l}$. На пространстве $H_{D}^{2 l}$ введем норму, полагая

$$
\|\varphi\|_{H_{D}^{2 l}}^{2}=\sum_{m \in \mathbf{Z}^{d}}\left|B_{m}\right|^{2}\|m\|^{4 l} .
$$

Легко показать, что $H_{D}^{2 l}$ является замкнутым подпространством $W_{2}^{2 l}(D)$, более того, норма $\|\cdot\|_{H_{D}^{2 l}}$ эквивалентна на этом подпространстве норме $\|\cdot\|_{W_{2}^{2 l}(D)}$.

Справедливо следующее утверждение.

Теорема 2. Пусть $\varphi \in H_{D}^{2 l+4}, l \geqslant 0, M(n)=n^{1 / 4}$. Тогда

$$
\sup _{t \in[0, T]}\left\|P_{n}^{t} \varphi-P^{t} \varphi\right\|_{W_{2}^{2 l}(D)} \leqslant C \frac{\|\varphi\|_{W_{2}^{2 l+4}(D)}}{n},
$$

где $C=C(l, d, T)$.

Д о к а з а т ель с т в о. Непосредственным вычислением получаем, что для каждого $m \in \mathbf{Z}^{d}$

$$
\mathbf{E} e^{i\left(m, \sigma \zeta_{n}(t)\right)}=\exp \left\{n t\left(\int_{\mathbf{R}^{d}} e^{i \sigma(m, y) / \sqrt{n}} d \mathscr{P}(y)-1\right)\right\} .
$$

Из (18), (19) и (20) следует, что

$$
P_{n}^{t} \varphi(x)=\sum_{\|m\| \leqslant M} B_{m} \exp \left\{n t\left(\int_{\mathbf{R}^{d}} e^{i \sigma(m, y) / \sqrt{n}} d \mathscr{P}(y)-1\right)\right\} e^{i(m, x)} .
$$

Далее, имеем

$$
\begin{aligned}
& \left\|P_{n}^{t} \varphi-P^{t} \varphi\right\|_{W_{2}^{2 l}}^{2} \\
& \leqslant C \sum_{\|m\| \leqslant M}\|m\|^{4 l}\left|B_{m}\right|^{2} \mid \exp \left\{n t\left(\int_{\mathbf{R}^{d}} e^{i \sigma(m, y) / \sqrt{n}} d \mathscr{P}(y)-1\right)\right\} \\
& \quad-\left.\exp \left\{-\frac{\sigma^{2}\|m\|^{2} t}{2}\right\}\right|^{2} \\
& \quad+C \sum_{\|m\|>M}\|m\|^{4 l}\left|B_{m}\right|^{2}\left|\exp \left\{-\frac{\sigma^{2}\|m\|^{2} t}{2}\right\}\right|^{2} \\
& =A_{1}+A_{2},
\end{aligned}
$$

где $A_{1}$ и $A_{2}$ обозначают соответственно первую и вторую суммы в правой части неравенства (22).

Оценим сначала слагаемое $A_{2}$. Используя (17), получим

$$
\begin{aligned}
A_{2} & \leqslant C \sum_{\|m\|>M}\|m\|^{4 l}\left|B_{m}\right|^{2} \\
& \leqslant \frac{C}{M^{8}} \sum_{\|m\|>M}\|m\|^{4 l+8}\left|B_{m}\right|^{2} \leqslant C \frac{\|\varphi\|_{W_{2}^{2 l+4}}^{2}}{M^{8}}=C \frac{\|\varphi\|_{W_{2}^{2 l+4}}^{2}}{n^{2}} .
\end{aligned}
$$


Теперь оценим слагаемое $A_{1}$. Заметим прежде всего, что суммирование в $A_{1}$ ведется по множеству $\|m\| \leqslant M$ и, тем самым, справедливо неравенство

$$
\frac{\|m\|}{\sqrt{n}} \leqslant \frac{M}{\sqrt{n}} \leqslant \frac{1}{n^{1 / 4}} .
$$

Напомним, что по условию случайная величина $\xi_{1}$ имеет единичную матрицу ковариаций, поэтому, снова используя (17), получим

$$
\begin{aligned}
\mid \exp & \left\{n t\left(\int_{\mathbf{R}^{d}} e^{i \sigma(p, m) / \sqrt{n}} d \mathscr{P}(y)-1\right)\right\}-\exp \left\{-\frac{\sigma^{2}\|m\|^{2} t}{2}\right\} \mid \\
\leqslant & \exp \left\{n t\left(\int_{\mathbf{R}^{d}} e^{i \sigma(m, y) / \sqrt{n}} d \mathscr{P}(y)-1+\frac{\sigma^{2}\|m\|^{2}}{2 n}\right)\right\}-1 \mid \\
& =\left|\exp \left\{n t \int_{\mathbf{R}^{d}}\left(e^{i \sigma(m, y) / \sqrt{n}}-1+\frac{\sigma^{2}(m, y)^{2}}{2 n}\right) d \mathscr{P}(y)\right\}-1\right| .
\end{aligned}
$$

Представим теперь интеграл, стоящий под экспонентой в правой части (25), в виде суммы двух интегралов - по области $\|y\| \leqslant n^{1 / 4}$ и по дополнительной области $\|y\|>n^{1 / 4}$, после чего воспользуемся элементарным неравенством

$$
|a b-1| \leqslant|a-1| \cdot|b|+|b-1| \text {. }
$$

Получим

$$
\begin{aligned}
\exp \left\{n t \int_{\mathbf{R}^{d}}\left(e^{i \sigma(m, y) / \sqrt{n}}-1+\frac{\sigma^{2}(m, y)^{2}}{2 n}\right) d \mathscr{P}(y)\right\}-1 \mid \\
\leqslant\left|\exp \left\{n t \int_{\|y\| \leqslant n^{1 / 4}}\left(e^{i \sigma(m, y) / \sqrt{n}}-1+\frac{\sigma^{2}(m, y)^{2}}{2 n}\right) d \mathscr{P}(y)\right\}-1\right| \\
\times\left|\exp \left\{n t \int_{\|y\|>n^{1 / 4}}\left(e^{i \sigma(m, y) / \sqrt{n}}-1+\frac{\sigma^{2}(m, y)^{2}}{2 n}\right) d \mathscr{P}(y)\right\}\right| \\
+\left|\exp \left\{n t \int_{\|y\|>n^{1 / 4}}\left(e^{i \sigma(m, y) / \sqrt{n}}-1+\frac{\sigma^{2}(m, y)^{2}}{2 n}\right) d \mathscr{P}(y)\right\}-1\right| .
\end{aligned}
$$

Интеграл

$$
\int_{\|y\|>n^{1 / 4}}\left(e^{i \sigma(m, y) / \sqrt{n}}-1+\frac{\sigma^{2}(m, y)^{2}}{2 n}\right) d \mathscr{P}(y)
$$

легко оценивается, именно, из условия (11) и неравенства (24) следует, что для любого $N>0$ найдется константа $C>0$ такая, что

$$
\left|\int_{\|y\|>n^{1 / 4}}\left(e^{i \sigma(m, y) / \sqrt{n}}-1+\frac{\sigma^{2}(m, y)^{2}}{2 n}\right) d \mathscr{P}(y)\right| \leqslant C n^{-N} .
$$


Оценим теперь интеграл

$$
\int_{\|y\| \leqslant n^{1 / 4}}\left(e^{i \sigma(m, y) / \sqrt{n}}-1+\frac{\sigma^{2}(m, y)^{2}}{2 n}\right) d \mathscr{P}(y) .
$$

Так как по условию случайная величина $\xi_{1}$ имеет симметричное распределение, то мы имеем

$$
\begin{array}{r}
\int_{\|y\| \leqslant n^{1 / 4}}\left(e^{i \sigma(m, y) / \sqrt{n}}-1+\frac{\sigma^{2}(m, y)^{2}}{2 n}\right) d \mathscr{P}(y) \\
=\int_{\|y\| \leqslant n^{1 / 4}}\left(e^{i \sigma(m, y) / \sqrt{n}}-1-\frac{i \sigma(m, y)}{\sqrt{n}}\right. \\
\left.+\frac{\sigma^{2}(m, y)^{2}}{2 n}+\frac{i \sigma^{3}(m, y)^{3}}{6 n^{3 / 2}}\right) d \mathscr{P}(y) .
\end{array}
$$

В области $\|y\| \leqslant n^{1 / 4}$ в силу (24) справедливо неравенство

$$
\left|e^{i \sigma(m, y) / \sqrt{n}}-1-\frac{i \sigma(m, y)}{\sqrt{n}}+\frac{\sigma^{2}(m, y)^{2}}{2 n}+\frac{i \sigma^{3}(m, y)^{3}}{6 n^{3 / 2}}\right| \leqslant C \frac{\|m\|^{4}\|y\|^{4}}{n^{2}} \text {. }
$$

Теперь, комбинируя (25), (27) и (29), получаем оценку

$$
\left|\exp \left\{n t\left(\int_{\mathbf{R}^{d}} e^{i \sigma(m, y) / \sqrt{n}} d \mathscr{P}(y)-1\right)\right\}-e^{-\sigma^{2}\|m\|^{2} t / 2}\right| \leqslant C \frac{\|m\|^{4}}{n} .
$$

Далее, используя (30), получаем

$$
\begin{gathered}
A_{1}=C \sum_{\|m\| \leqslant M}\|m\|^{4 l}\left|B_{m}\right|^{2} \mid \exp \left\{n t\left(\int_{\mathbf{R}^{d}} e^{i \sigma(m, y) / \sqrt{n}} d \mathscr{P}(y)-1\right)\right\} \\
-\left.e^{-\sigma^{2}\|m\|^{2} t / 2}\right|^{2} \\
\leqslant \frac{C}{n^{2}} \sum_{\|m\| \leqslant M}\|m\|^{4 l+8}\left|B_{m}\right|^{2} \leqslant \frac{C}{n^{2}}\|\varphi\|_{W_{2}^{2 l+4} .}^{2}
\end{gathered}
$$

Наконец, из последней оценки, (22) и (23) следует утверждение теоремы.

4. Задача Дирихле в ограниченной области с гладкой границей. Пусть $D_{0}$ - ограниченная область в $\mathbf{R}^{d}, d \geqslant 2$, с границей $\partial D_{0}$, принадлежащей классу $C^{\infty}$.

Выберем такой куб $D$, что его внутренность содержит замыкание множества $D_{0}$. Как и выше, для простоты мы предположим, что $D=$ $[0, \pi]^{d}$.

Пусть $0<\lambda_{1} \leqslant \lambda_{2} \leqslant \cdots$ - собственные значения, а $s_{1}(x), s_{2}(x), \ldots-$ соответствующие собственные функции оператора $-\Delta$ в $D_{0}$ с нулевыми 
граничными условиями (именно, самосопряженного оператора $-\Delta_{D_{0}}$, действующего в пространстве $L_{2}\left(D_{0}\right)$ и заданного на области определения $\left.W_{2}^{2,0}\left(D_{0}\right)\right)$. Это значит, что для каждого $j=1,2, \ldots$ функция $s_{j}$ является решением начально-краевой задачи (с $\left.\lambda=\lambda_{j}\right)$

$$
\Delta u+\lambda u=0,\left.\quad u\right|_{\partial D_{0}}=0
$$

Нормируем функции $s_{j}$ так, что для каждого $j \in \mathbf{N}$

$$
\left\|s_{j}\right\|_{L_{2}\left(D_{0}\right)}=1
$$

Известна асимптотика собственных значений $\lambda_{j}$ при $j \rightarrow \infty$, полученная Г. Вейлем (см. [15, с. 205, формула (17.3.6)]). Именно, существует такая константа $C$, что

$$
\lambda_{j} \sim C j^{2 / d}, \quad j \rightarrow \infty
$$

Собственные функции $\left\{s_{j}\right\}$ образуют ортонормированный базис в $L_{2}\left(D_{0}\right)$, и, тем самым, всякая функция $\varphi \in L_{2}\left(D_{0}\right)$ раскладывается в ряд (сходящийся по норме $L_{2}\left(D_{0}\right)$ )

$$
\varphi(x)=\sum_{j=1}^{\infty} a_{j} s_{j}(x) .
$$

Для $l \in \mathbf{N}$ через $H_{D_{0}}^{2 l}$ обозначим множество функций $\varphi: D_{0} \rightarrow \mathbf{C}$ таких, что сходится ряд $\sum_{j=1}^{\infty}\left|a_{j}\right|^{2} j^{4 l / d}$.

На пространстве $H_{D_{0}}^{2 l}$ введем норму, полагая

$$
\|\varphi\|_{H_{D_{0}}^{2 l}}^{2}=\sum_{j=1}^{\infty}\left|a_{j}\right|^{2} j^{4 l / d} .
$$

Покажем, что на $H_{D_{0}}^{2 l}$ норма $\|\cdot\|_{H_{D_{0}}^{2 l}}$ эквивалентна соболевской норме $\|\cdot\|_{W_{2}^{2 l}\left(D_{0}\right)} \cdot$

Лемма 1. Существуют такие положительнье константь $C_{1}=$ $C_{1}\left(D_{0}\right), C_{2}=C_{2}\left(D_{0}\right)$, что для каждого $\varphi \in H_{D_{0}}^{2 l}$

$$
C_{1}\|\varphi\|_{W_{2}^{2 l}\left(D_{0}\right)} \leqslant\|\varphi\|_{H_{D_{0}}^{2 l}} \leqslant C_{2}\|\varphi\|_{W_{2}^{2 l}\left(D_{0}\right)} .
$$

Д о к а з а т е л ь с т в о. В силу (32) имеем

$$
\begin{aligned}
\|\varphi\|_{H_{D_{0}}^{2 l}} & =\left(\sum_{j=1}^{\infty}\left|a_{j}\right|^{2} j^{4 l / d}\right)^{1 / 2} \leqslant C\left(\sum_{j=1}^{\infty}\left|a_{j}\right|^{2} \lambda_{j}^{2 l}\right)^{1 / 2} \\
& =C\left\|\Delta^{l} \varphi\right\|_{L_{2}\left(D_{0}\right)} \leqslant C\|\varphi\|_{W_{2}^{2 l}\left(D_{0}\right)} .
\end{aligned}
$$


Таким образом, второе неравенство доказано. Для доказательства первого неравенства воспользуемся теоремой 5.1 из [14, гл. 2] (см. также [20, теорема 7.1.3]) о повышении гладкости решений уравнения эллиптического типа в области. Применительно к нашей задаче указанные теоремы означают справедливость оценки $\|\varphi\|_{W_{2}^{2 l}\left(D_{0}\right)} \leqslant C\left(\left\|\Delta^{l} \varphi\right\|_{L_{2}\left(D_{0}\right)}+\right.$ $\left.\|\varphi\|_{L_{2}\left(D_{0}\right)}\right)$. Поэтому

$$
\|\varphi\|_{W_{2}^{2 l}\left(D_{0}\right)} \leqslant C\left(\sum_{j=1}^{\infty}\left(\lambda_{j}^{2 l}+1\right)\left|a_{j}\right|^{2}\right)^{1 / 2} \leqslant C\left(\sum_{j=1}^{\infty} j^{4 l / d}\left|a_{j}\right|^{2}\right)^{1 / 2} .
$$

Лемма доказана.

Из леммы 1 вытекает, что $H_{D_{0}}^{2 l}$ является замкнутым подпространством $W_{2}^{2 l}\left(D_{0}\right)$. Заметим, что в случае, когда число $l$ достаточно большое, именно $4 l>d$, в силу классической теоремы вложения Соболева (см., например, [18], [19]) оператор вложения $W_{2}^{2 l}\left(D_{0}\right) \subset C\left(D_{0}\right)$ ограничен, так что все функции из $H_{D_{0}}^{2 l}$ непрерывны.

Как и выше, для $t \geqslant 0$ через $P_{0}^{t}$ обозначим операторную экспоненту $e^{\left(t \sigma^{2} / 2\right) \Delta_{D_{0}}}$. Используя метод разделения переменных, нетрудно показать, что оператор $P_{0}^{t}$ действует на функцию $\varphi(x)=\sum_{j=1}^{\infty} a_{j} s_{j}(x)$ следующим образом:

$$
P_{0}^{t} \varphi(x)=\sum_{j=1}^{\infty} a_{j} e^{-\sigma^{2} t \lambda_{j} / 2} s_{j}(x),
$$

другими словами, функция $u(t, x)=P_{0}^{t} \varphi(x)$ есть единственное решение уравнения (1) с начально-краевыми условиями $u(0, x)=\varphi(x),\left.u\right|_{\partial D_{0}}=0$. При этом из условия $\operatorname{Re} \sigma^{2} \geqslant 0$ вытекает, что при всех $j \in \mathbf{N}$ выполнено

$$
\left|e^{-\sigma^{2} t \lambda_{j} / 2}\right| \leqslant 1 \text {. }
$$

Как известно (см., например, $[17$, гл. 6], $[19,4.3, \S 10])$, всякую функцию, принадлежащую пространству $W_{2}^{k}\left(D_{0}\right)$ в области $D_{0}$ с гладкой границей, можно продолжить за пределы этой области с сохранением класса. Именно, если замыкание области $D_{0}$ содержится во внутренности области $D_{1}$, то существует ограниченный оператор $\Pi: W_{2}^{k}\left(D_{0}\right) \rightarrow$ $\stackrel{\circ}{W}_{2}^{k}\left(D_{1}\right)$ такой, что $(\Pi u)(x)=u(x)$ при всех $u \in W_{2}^{k}\left(D_{0}\right), x \in D_{0}$. Введем в наши рассмотрения оператор продолжения (здесь $D=[0, \pi]^{d}$ )

$$
\Pi: W_{2}^{2 l+4}\left(D_{0}\right) \rightarrow \stackrel{\circ}{W_{2}^{2 l+4}}(D) .
$$

Пусть $\mathscr{H}_{M}$, как и выше, обозначает оператор проектирования (18), а $\widetilde{\mathscr{H}}_{J}$ обозначает проектор в $L_{2}\left(D_{0}\right)$ на линейную оболочку $\mathscr{L}\left(s_{1}, \ldots, s_{J}\right)$. Далее у нас $M$ и $J$ будут зависеть от $n: M=M(n), J=J(n)$.

Введем еще одно обозначение. Для каждой функции $\varphi \in L_{2}\left(D_{0}\right)$ через $\varphi_{M, J}$ будем обозначать функцию (тригонометрический полином)

$$
\varphi_{M, J}(x)=\mathscr{H}_{M}\left(\Pi \widetilde{\mathscr{H}}_{J} \varphi\right)^{\text {odd }}(x),
$$


т.е. сначала мы проектируем $\varphi$ на $\mathscr{L}\left(s_{1}, \ldots, s_{J}\right)$ (получившаяся функция автоматически оказывается принадлежащей пространству $\left.W_{2}^{2 l+4}\left(D_{0}\right)\right)$, затем продолжаем получившуюся функцию оператором продолжения П на $D=[0, \pi]^{d}$, продолжаем нечетным образом на $[-\pi, \pi]^{d}$ и, наконец, проектируем на $\mathscr{L}\left(e_{m},\|m\| \leqslant M\right)$.

Определим теперь семейство операторов (не полугруппу) $Q_{n}^{t}, t \geqslant 0$, полагая для $\varphi \in L_{2}\left(D_{0}\right), x \in D_{0}$

$$
Q_{n}^{t} \varphi(x)=\mathbf{E} \varphi_{M, J}\left(x+\sigma \zeta_{n}(t)\right) .
$$

Справедливо следующее утверждение.

Теорема 3. Пусть $\varphi \in H_{D_{0}}^{2 l+4}, l \geqslant 0, J(n)=n^{2 d / d+16}, M(n)=\varepsilon_{0} n^{1 / 2}$, где $\varepsilon_{0}$ - произвольное положительное число, удовлетворяющее условию $|\sigma| \varepsilon_{0}<\gamma$. Тогда

$$
\sup _{t \in[0, T]}\left\|\left(Q_{n}^{t / n}\right)^{n} \varphi-P_{0}^{t} \varphi\right\|_{W_{2}^{2 l}\left(D_{0}\right)} \leqslant C \frac{\|\varphi\|_{W_{2}^{2 l+4}\left(D_{0}\right)}}{n^{1 /(2+d / 8)}},
$$

әде $C=C\left(l, d, T, D_{0}\right)$.

Д о к а з а т е л ь с т в о. Посмотрим сначала на продолжения собственных функций. Пусть П $s_{j}$ - продолжение собственной функции $s_{j}$. Как и выше, $\left(\Pi s_{j}\right)^{\text {odd }}$ будет обозначать нечетное продолжение функции $\Pi s_{j}$ с $D=[0, \pi]^{d}$ на $[-\pi, \pi]^{d}$. Разложим функцию $\left(\Pi s_{j}\right)^{\text {odd }}$ в ряд Фурье:

$$
\left(\Pi s_{j}\right)^{\text {odd }}(x)=\sum_{m \in \mathbf{Z}^{d}} K_{m}^{j} e^{i(m, x)} .
$$

Лемма 2. Cуществует $C=C(d, l)>0$ maкое, ито

$$
\left(\sum_{m \in \mathbf{Z}}\left|K_{m}^{j}\right|^{2}\|m\|^{4 l+8}\right)^{1 / 2} \leqslant C j^{(2 l+4) / d} .
$$

Д о к а з а т е л с т в о. Имеем

$$
\left(\sum_{m \in \mathbf{Z}}\left|K_{m}^{j}\right|^{2}\|m\|^{4 l+8}\right)^{1 / 2}=C\left\|\Delta^{l+2} \Pi s_{j}\right\|_{L_{2}(D)} \leqslant C\left\|\Pi s_{j}\right\|_{W_{2}^{2 l+4}(D)} .
$$

Воспользуемся непрерывностью оператора продолжения и теоремой 5.1 из [14, гл. 2]. Получим

$$
\begin{aligned}
\left\|\Pi s_{j}\right\|_{W_{2}^{2 l+4}(D)} & \leqslant C\left\|s_{j}\right\|_{W_{2}^{2 l+4}\left(D_{0}\right)} \leqslant C\left\|\Delta^{l+2} s_{j}\right\|_{L_{2}\left(D_{0}\right)} \\
& \leqslant C \lambda_{j}^{l+2} \leqslant C j^{(2 l+4) / d}
\end{aligned}
$$

что и доказывает лемму. 
Итак, пусть $\varphi=\sum_{j=1}^{\infty} a_{j} s_{j}$. Тогда $\widetilde{\mathscr{H}}_{J} \varphi=\sum_{j=1}^{J} a_{j} s_{j}$ и в силу $(40)$ для $x \in D$ выполнено

$$
\Pi \widetilde{\mathscr{H}}_{J} \varphi(x)=\sum_{j=1}^{J} a_{j}\left(\sum_{m \in \mathbf{Z}^{d}} K_{m}^{j} e^{i(m, x)}\right) .
$$

Заметим, что на $D_{0}$ функции $\widetilde{\mathscr{H}}_{J} \varphi$ и П $\widetilde{\mathscr{H}}_{J} \varphi$ совпадают, значит, для $x \in D_{0}$ мы имеем

$$
\sum_{j=1}^{J} a_{j} s_{j}(x)=\sum_{j=1}^{J} a_{j}\left(\sum_{m \in \mathbf{Z}^{d}} K_{m}^{j} e^{i(m, x)}\right),
$$

а применяя оператор Лапласа к левой и правой частям (42), получаем, что для $x \in D_{0}$ также справедливо

$$
\sum_{j=1}^{J} a_{j} \lambda_{j} s_{j}(x)=\sum_{j=1}^{J} a_{j}\left(\sum_{m \in \mathbf{Z}^{d}} K_{m}^{j}\|m\|^{2} e^{i(m, x)}\right) .
$$

Далее, в силу (41) имеем

$$
\varphi_{M, J}(x)=\mathscr{H}_{M}\left(\Pi \widetilde{\mathscr{H}}_{J} \varphi\right)^{\text {odd }}(x)=\sum_{j=1}^{J} a_{j}\left(\sum_{\|m\| \leqslant M} K_{m}^{j} e^{i(m, x)}\right) .
$$

Теперь, используя (20), получаем

$$
\begin{aligned}
& Q_{n}^{t / n} \varphi(x)= \mathbf{E} \varphi_{M, J}\left(x+\sigma \zeta_{n}\left(\frac{t}{n}\right)\right) \\
&= \sum_{j=1}^{J} a_{j}\left(\sum_{\|m\| \leqslant M} K_{m}^{j} \exp \left\{t\left(\int_{\mathbf{R}^{d}} e^{i \sigma(m, y) / \sqrt{n}} d \mathscr{P}(y)-1\right)\right\} e^{i(m, x)}\right) \\
&= \sum_{j=1}^{J} a_{j}\left(\sum_{\|m\| \leqslant M} K_{m}^{j} e^{i(m, x)}\right)-\frac{\sigma^{2} t}{2 n} \sum_{j=1}^{J} a_{j}\left(\sum_{\|m\| \leqslant M}\|m\|^{2} K_{m}^{j} e^{i(m, x)}\right) \\
&+\sum_{j=1}^{J} a_{j}\left(\sum _ { \| m \| \leqslant M } \left[K_{m}^{j} \exp \left\{t\left(\int_{\mathbf{R}^{d}} e^{i \sigma(m, y) / \sqrt{n}} d \mathscr{P}(y)-1\right)\right\}\right.\right. \\
&\left.\left.-1+\frac{\sigma^{2} t\|m\|^{2}}{2 n}\right] e^{i(m, x)}\right) .
\end{aligned}
$$

Из последнего равенства и соотношений (41), (42) следует, что

$$
\begin{aligned}
Q_{n}^{t / n} \varphi(x)= & \sum_{j=1}^{J} a_{j} s_{j}(x)-\frac{\sigma^{2} t}{2 n} \sum_{j=1}^{J} a_{j} \lambda_{j} s_{j}(x)-\sum_{j=1}^{J} a_{j}\left(\sum_{\|m\|>M} K_{m}^{j} e^{i(m, x)}\right) \\
& +\frac{\sigma^{2} t}{2 n} \sum_{j=1}^{J} a_{j}\left(\sum_{\|m\|>M}\|m\|^{2} K_{m}^{j} e^{i(m, x)}\right)
\end{aligned}
$$




$$
\begin{gathered}
+\sum_{j=1}^{J} a_{j}\left(\sum _ { \| m \| \leqslant M } K _ { m } ^ { j } \left[\exp \left\{t\left(\int_{\mathbf{R}^{d}} e^{i \sigma(m, y) / \sqrt{n}} d \mathscr{P}(y)-1\right)\right\}\right.\right. \\
\left.\left.-1+\frac{\sigma^{2} t\|m\|^{2}}{2 n}\right] e^{i(m, x)}\right)
\end{gathered}
$$

Таким образом,

$$
Q_{n}^{t / n} \varphi(x)=\sum_{j=1}^{J} a_{j}\left(1-\frac{\sigma^{2} t \lambda_{j}}{2 n}\right) s_{j}(x)+A_{1}(x)+A_{2}(x)+A_{3}(x),
$$

где $A_{1}, A_{2}, A_{3}$ обозначают соответственно три последних слагаемых в (44). Следующим шагом оценим $W_{2}^{2 l}\left(D_{0}\right)$-норму слагаемых $A_{1}, A_{2}, A_{3}$. Имеем

$$
-A_{1}(x)=\sum_{j=1}^{J} a_{j} b_{j}^{1}(x), \quad \text { где } b_{j}^{1}(x)=\sum_{\|m\|>M} K_{m}^{j} e^{i(m, x)} .
$$

При этом

$$
\begin{aligned}
\left\|b_{j}^{1}\right\|_{W_{2}^{2 l}\left(D_{0}\right)} & \leqslant\left\|b_{j}^{1}\right\|_{W_{2}^{2 l}\left([-\pi, \pi]^{d}\right)}=C\left(\sum_{\|m\|>M}\left|K_{m}^{j}\right|^{2}\|m\|^{4 l}\right)^{1 / 2} \\
& \leqslant \frac{C}{M^{4}}\left(\sum_{\|m\|>M}\left|K_{m}^{j}\right|^{2}\|m\|^{4 l+8}\right)^{1 / 2} \\
& \leqslant \frac{C}{n^{2}}\left\|s_{j}\right\|_{W_{2}^{2 l+4}\left(D_{0}\right)} \leqslant \frac{C j^{(2 l+4) / d}}{n^{2}}
\end{aligned}
$$

(на последнем шаге мы воспользовались оценкой леммы 2).

Далее, имеем $A_{2}(x)=\sum_{j=1}^{J} a_{j} b_{j}^{2}(x)$, где

$$
b_{j}^{2}(x)=\frac{\sigma^{2} t}{2 n} \sum_{\|m\|>M}\|m\|^{2} K_{m}^{j} e^{i(m, x)} .
$$

Аналогичные рассуждения приводят нас к оценке

$$
\left\|b_{j}^{2}\right\|_{W_{2}^{2 l}\left(D_{0}\right)} \leqslant \frac{C j^{(2 l+4) / d}}{n^{2}} .
$$

Наконец, мы имеем $A_{3}(x)=\sum_{j=1}^{J} a_{j} b_{j}^{3}(x)$, где

$$
\begin{gathered}
b_{j}^{3}(x)=\sum_{\|m\| \leqslant M} K_{m}^{j}\left[\exp \left\{t\left(\int_{\mathbf{R}^{d}} e^{i \sigma(m, y) / \sqrt{n}} d \mathscr{P}(y)-1\right)\right\}\right. \\
\left.-1+\frac{\sigma^{2} t\|m\|^{2}}{2 n}\right] e^{i(m, x)} .
\end{gathered}
$$

Соответствующая оценка здесь менее очевидна, мы сформулируем ее в виде отдельной леммы. 
Лемма 3. Для некоторого $C>0$ справедлива оченка

$$
\left\|b_{j}^{3}\right\|_{W_{2}^{2 l}\left(D_{0}\right)} \leqslant \frac{C j^{(2 l+4) / d}}{n^{2}} .
$$

Д о к а з а т е л ь с т в о. Оценим сначала выражение, стоящее в (47) в квадратных скобках. Имеем

$$
\begin{aligned}
& \left|\exp \left\{t\left(\int_{\mathbf{R}^{d}} e^{i \sigma(m, y) / \sqrt{n}} d \mathscr{P}(y)-1\right)\right\}-1+\frac{\sigma^{2} t\|m\|^{2}}{2 n}\right| \\
& \leqslant\left|\exp \left\{t\left(\int_{\mathbf{R}^{d}} e^{i \sigma(m, y) / \sqrt{n}} d \mathscr{P}(y)-1\right)\right\}-\exp \left\{-\frac{\sigma^{2} t\|m\|^{2}}{2 n}\right\}\right| \\
& \quad+\left|\exp \left\{-\frac{\sigma^{2} t\|m\|^{2}}{2 n}\right\}-1+\frac{\sigma^{2} t\|m\|^{2}}{2 n}\right| .
\end{aligned}
$$

Так как у нас по условию $\|m\|^{2} / n \leqslant \varepsilon_{0}$, то второе слагаемое в правой части (48) оценивается сверху величиной $C\|m\|^{4} / n^{2}$. Оценим теперь первое слагаемое. Имеем

$$
\begin{aligned}
& \left|\exp \left\{t\left(\int_{\mathbf{R}^{d}} e^{i \sigma(m, y) / \sqrt{n}} d \mathscr{P}(y)-1\right)\right\}-\exp \left\{-\frac{\sigma^{2} t\|m\|^{2}}{2 n}\right\}\right| \\
& \leqslant\left|\exp \left\{t\left(\int_{\mathbf{R}^{d}}\left(e^{i \sigma(m, y) / \sqrt{n}}-1+\frac{\sigma^{2}(m, y)^{2}}{2 n}\right) d \mathscr{P}(y)\right)\right\}-1\right| .
\end{aligned}
$$

Оценим теперь интеграл, стоящий под знаком экспоненты в правой части формулы (49). Так как по условию распределение $\mathscr{P}$ симметрично, а $|\sigma| \varepsilon_{0}<\gamma$, то мы имеем

$$
\begin{aligned}
\mid \int_{\mathbf{R}^{d}} & \left(e^{i \sigma(m, y) / \sqrt{n}}-1+\frac{\sigma^{2}(m, y)^{2}}{2 n}\right) d \mathscr{P}(y) \mid \\
\quad= & \left|\int_{\mathbf{R}^{d}}\left(e^{i \sigma(m, y) / \sqrt{n}}-1-\frac{i \sigma(m, y)}{\sqrt{n}}+\frac{\sigma^{2}(m, y)^{2}}{2 n}+\frac{i \sigma^{3}(m, y)^{3}}{6 n^{3 / 2}}\right) d \mathscr{P}(y)\right| \\
\quad \leqslant C & \frac{\|m\|^{4}}{n^{2}} .
\end{aligned}
$$

Из последней оценки немедленно следует, что первое слагаемое в правой части (48) также оценивается сверху величиной $C\|m\|^{4} / n^{2}$. Далее, рассуждая также, как при доказательстве неравенства (45), получаем утверждение леммы.

Из (45), (46) и леммы 3 следует, что $A_{1}+A_{2}+A_{3}=\sum_{j=1}^{J} a_{j} b_{j}$, причем

$$
\left\|b_{j}\right\|_{W_{2}^{2 l}\left(D_{0}\right)} \leqslant \frac{C j^{(2 l+4) / d}}{n^{2}}
$$


и, значит,

$$
\begin{gathered}
\left\|A_{1}+A_{2}+A_{3}\right\|_{W_{2}^{2 l}\left(D_{0}\right)} \leqslant \frac{C}{n^{2}} \sum_{j=1}^{J}\left|a_{j}\right| j^{(2 l+4) / d} \leqslant \frac{C J^{1 / 2+4 / d}}{n^{2}}\left(\sum_{j=1}^{J}\left|a_{j}\right|^{2} j^{4 l / d}\right)^{1 / 2} \\
\leqslant \frac{C J^{1 / 2+4 / d}}{n^{2}}\|\varphi\|_{W_{2}^{2 l}\left(D_{0}\right)} \\
=\frac{C}{n^{1+8 /(d+16)}}\|\varphi\|_{W_{2}^{2 l}\left(D_{0}\right)} .
\end{gathered}
$$

Таким образом, мы получили представление оператора $Q_{n}^{t / n}$ в виде

$$
Q_{n}^{t / n}=G_{n}+R_{n}
$$

где $G_{n}$ - диагональный (в базисе $\left\{s_{j}\right\}$ ) оператор вида

$$
G_{n} \varphi(x)=\sum_{j=1}^{J} a_{j}\left(1-\frac{\sigma^{2} t \lambda_{j}}{2 n}\right) s_{j}(x)
$$

а для операторной нормы оператора $R_{n}: W_{2}^{2 l}\left(D_{0}\right) \rightarrow W_{2}^{2 l}\left(D_{0}\right)$ в силу (50) мы имеем оценку

$$
\left\|R_{n}\right\|_{W_{2}^{2 l}\left(D_{0}\right) \rightarrow W_{2}^{2 l}\left(D_{0}\right)} \leqslant \frac{C}{n^{1+8 /(d+16)}} .
$$

Далее, в силу (51) имеем

$$
\begin{aligned}
\left\|\left(Q_{n}^{t / n}\right)^{n} \varphi-P_{0}^{t} \varphi\right\|_{W_{2}^{2 l}\left(D_{0}\right)} \leqslant & \left\|\left(G_{n}+R_{n}\right)^{n} \varphi-\sum_{j=1}^{J} a_{j} e^{-\sigma^{2} t \lambda_{j} / 2} s_{j}\right\|_{W_{2}^{2 l}\left(D_{0}\right)} \\
& +\left\|\sum_{j=J+1}^{\infty} a_{j} e^{-\sigma^{2} t \lambda_{j} / 2} s_{j}\right\|_{W_{2}^{2 l}\left(D_{0}\right)} \\
\leqslant & \left\|\left(G_{n}\right)^{n} \varphi-\sum_{j=1}^{J} a_{j} e^{-\sigma^{2} t \lambda_{j} / 2} s_{j}\right\|_{W_{2}^{2 l}\left(D_{0}\right)} \\
& +\left\|\sum_{k=1}^{n} T_{k, n} \varphi\right\|_{W_{2}^{2 l}\left(D_{0}\right)} \\
& +\left\|\sum_{j=J+1}^{\infty} a_{j} e^{-\sigma^{2} t \lambda_{j} / 2} s_{j}\right\|_{W_{2}^{2 l}\left(D_{0}\right)}
\end{aligned}
$$

где $T_{k, n}$ обозначает сумму всевозможных произведений операторов $G_{n}$ и $R_{n}$, содержащих $R_{n}$ ровно $k$ раз. Обозначим теперь через $V_{1}, V_{2}, V_{3}$ соответственно первое, второе и третье слагаемые в правой части (54). Оценим сначала $V_{3}$. Имеем

$$
V_{3} \leqslant\left(\sum_{j=J+1}^{\infty}\left|a_{j}\right|^{2} j^{4 l / d}\right)^{1 / 2} \leqslant \frac{C}{J^{4 / d}}\left(\sum_{j=J+1}^{\infty}\left|a_{j}\right|^{2} j^{(4 l+8) / d}\right)^{1 / 2} \leqslant \frac{C\|\varphi\|_{W_{2}^{2 l+4}\left(D_{0}\right)}}{n^{1 /(2+d / 8)}} .
$$


Оценим теперь $V_{2}$. Операторная норма (из $W_{2}^{2 l}\left(D_{0}\right)$ в $W_{2}^{2 l}\left(D_{0}\right)$ ) оператора $T_{k, n}$ оценивается сверху величиной

$$
\left(\begin{array}{l}
n \\
k
\end{array}\right)\left\|G_{n}\right\|^{n-k}\left\|R_{n}\right\|^{k}
$$

при этом из условия $\operatorname{Re} \sigma^{2} \geqslant 0$ следует, что $\left\|G_{n}\right\| \leqslant 1$. Таким образом, в силу (53)

$$
\begin{aligned}
V_{2} & =\left\|\sum_{k=1}^{n} T_{k, n} \varphi\right\|_{W_{2}^{2 l}\left(D_{0}\right)} \leqslant\|\varphi\|_{W_{2}^{2 l}\left(D_{0}\right)}\left\|\sum_{k=1}^{n} T_{k, n}\right\| \\
& \leqslant\|\varphi\|_{W_{2}^{2 l}\left(D_{0}\right)} \sum_{k=1}^{n}\left(\begin{array}{c}
n \\
k
\end{array}\right)\left\|R_{n}\right\|^{k}=\|\varphi\|_{W_{2}^{2 l}\left(D_{0}\right)}\left(\left(1+\left\|R_{n}\right\|\right)^{n}-1\right) \\
& =\|\varphi\|_{W_{2}^{2 l}\left(D_{0}\right)}\left(e^{n \ln \left(1+\left\|R_{n}\right\|\right)}-1\right) \leqslant\|\varphi\|_{W_{2}^{2 l}\left(D_{0}\right)}\left(e^{n\left\|R_{n}\right\|}-1\right) \\
& \leqslant C\|\varphi\|_{W_{2}^{2 l}\left(D_{0}\right)} \cdot n\left\|R_{n}\right\| \leqslant \frac{C\|\varphi\|_{W_{2}^{2 l}\left(D_{0}\right)}}{n^{1 /(2+d / 8)}} \leqslant \frac{C\|\varphi\|_{W_{2}^{2 l+4}\left(D_{0}\right)}}{n^{1 /(2+d / 8)}} .
\end{aligned}
$$

Осталось оценить слагаемое $V_{1}$. Используя неравенство $(n \geqslant 2)$

$$
\left|e^{-z}-\left(1-\frac{z}{n}\right)^{n}\right| \leqslant C \frac{|z|^{2}}{n},
$$

справедливое для комплексных $z$, удовлетворяющих условиям $\operatorname{Re} z \geqslant 0$, $|z|^{2} \leqslant n / 2$, получаем

$$
\begin{aligned}
V_{1} & =\left\|\sum_{j=1}^{J} a_{j}\left(1-\frac{\sigma^{2} t \lambda_{j}}{2 n}\right)^{n} s_{j}-\sum_{j=1}^{J} a_{j} e^{-\sigma^{2} t \lambda_{j} / 2} s_{j}\right\|_{W_{2}^{2 l}\left(D_{0}\right)} \\
& \leqslant C\left(\sum_{j=1}^{J}\left|a_{j}\right|^{2} j^{4 l / d}\left|e^{-\frac{\sigma^{2} t \lambda_{j}}{2}}-\left(1-\frac{\sigma^{2} t \lambda_{j}}{2 n}\right)^{n}\right|^{2}\right)^{1 / 2} \\
& \leqslant \frac{C}{n}\left(\sum_{j=1}^{J}\left|a_{j}\right|^{2} j^{4 l / d} \lambda_{j}^{4}\right)^{1 / 2} \leqslant \frac{C}{n}\|\varphi\|_{W_{2}^{2 l+4}\left(D_{0}\right)} .
\end{aligned}
$$

Теорема 3 доказана. 


\section{СПИСОК ЛИТЕРАТУРЫ}

1. Chung K. L., Zhao Z. From Brownian Motion to Schrodinger's Equation. Berlin: Springer-Verlag, 1995.

2. Freidlin M. Functional Integration and Partial Differential Equations. Princeton: Princeton Univ. Press, 1985, 545 p.

3. Далецкий Ю. Л., Фомин С. В. Меры и дифференциальные уравнения в функциональных пространствах. М.: Наука, 1983, 383 с.

4. Beghin L., Orsingher E. The distribution of the local time for «pseudoprocess» and its connection with fractional diffusion equatios. - Stochastic Process. Appl., 2005, v. 115 , № 6 , c. 1017-1040.

5. Lachal $A$. First hitting time and place for pseudo-processes driven by the equation $\partial / \partial t= \pm \partial^{N} / \partial x^{N}$ subject to a linear drift. - J. Stochastic Process. Appl., 2008, v. 118, № 1, p. 1-27.

6. Глимм Дж., Джаффе А. Математические методы квантовой физики. Подход с использованием функциональных интегралов. М.: Мир, 1984, 445 с.

7. Рид М., Саймон Б. Методы современной математической физики. М.: Мир, 1978.

8. Маслов В.П. Комплексные марковские цепи и континуальный интеграл Фейнмана. М.: Наука, 1976, 191 с.

9. Маслов В.П., Чеботарев А. М. Определение континуального интеграла Фейнмана в $p$-представлении. - Докл. АН СССР, 1976, т. 229, № 1, с. 37-38.

10. Чеботарев A.M. О представлении решения уравнения Шрёдингера в виде математического ожидания функционалов скачкообразного процесса. - Матем. заметки, 1978, т. 24, № 5, с. 699-706.

11. Ибрагимов И.А., Смородина Н.В., Фаддеев М. М. Вероятностный подход к построению решений одномерных начально-краевых задач. - Теория вероятн. и ее примен., 2013, т. 58, в. 2, с. 255-281.

12. Ibragimov I. A., Smorodina N.V., Faddeev M. M. The probabilistic approximation of the Dirichlet initial boundary value problem solution for the equation $\partial u / \partial t=\left(\sigma^{2} / 2\right) \Delta u$ with a complex parameter $\sigma$. - Markov Process. Related Fields (в печати).

13. Ибрагимов И.А., Смородина Н.В., Фаддеев М.М. Предельная теорема о сходимости функционалов от случайного блуждания к решению задачи Коши для уравнения $\partial u / \partial t=\left(\sigma^{2} / 2\right) \Delta u$ с комплексным параметром $\sigma$. - Зап. научн. сем. ПОМИ, 2013, т. 420, с. 88-102.

14. Лионс Ж.-Л., Мадженес Э. Неоднородные граничные задачи и их приложения. М.: Мир, 1971, 371 с.

15. Титчмари Э. Ч. Разложения по собственным функциям, связанные с дифференциальными уравнениями второго порядка, т. 2. М.: ИЛ, 1961, 555 с.

16. Ладыженская O.А., Уральщева Н. Н. Линейные и квазилинейные уравнения эллиптического типа. М.: Наука, 1973, 736 с.

17. Стейн И. М. Сингулярные интегралы и дифференциальные свойства функций. М.: Мир, 1973, 342 с.

18. Смирнов В. И. Курс высшей математики, т. 5. М.: Наука, 1959, 655 с.

19. Фаддеев Д. К., Вулих Б. З., Уральчева Н. Н. и др. Избранные главы анализа и высшей алгебры. Л.: Изд-во Ленингр. ун-та, 1981, 200 с.

20. Агранович M. С. Соболевские пространства, их обобщения и эллиптические задачи в областях с гладкой и липшицевой границей. М.: МЦНМО, 2013, 378 с. 